\title{
Enhanced Safety Surveillance of GSK's Quadrivalent Seasonal Influenza Vaccine in Belgium, Germany, and Spain for the 2018/19 Season: Interim Analysis
}

\author{
Gael Dos Santos · Vishvesh Shende - Silvia Damaso - Anne Yeakey
}

Received: August 23, 2019 / Published online: October 8, 2019

(C) The Author(s) 2019

\begin{abstract}
Introduction: Influenza is an important cause of morbidity and mortality in Europe. Prevention by annual vaccination is most effective but with yearly vaccine reformulation to match circulating virus strains, vaccine safety must be continuously monitored. The European Medicines Agency published guidance on safety monitoring of influenza vaccines.

Methods: An enhanced safety surveillance study of GSK's inactivated quadrivalent influenza vaccine (IIV4) was conducted in Belgium, Germany, and Spain in influenza season $2018 / 19$. The objective was to collect adverse event (AE) reports from subjects within 7 days of vaccination. A customized $\mathrm{AE}$ reporting card
\end{abstract}

Enhanced Digital Features To view enhanced digital features for this article go to https://doi.org/10.6084/ m9.figshare.9885899.

Electronic supplementary material The online version of this article (https://doi.org/10.1007/s12325019-01105-2) contains supplementary material, which is available to authorized users.

G. Dos Santos $(\varangle) \cdot$ S. Damaso

GSK, Wavre, Belgium

e-mail: gael.x.dos-santos@gsk.com

V. Shende

VPN Consultancy Ltd, London, UK

A. Yeakey

GSK, Rockville, MD, USA
(AERC) with predefined AEs of interest was used to rapidly detect and evaluate potential new safety concerns. Interim results are presented here.

Results: Between week 40 and 52, 1060 vaccinated subjects were enrolled (31.0\% Belgium, $26.2 \%$ Germany, and $42.7 \%$ Spain) covering all ages for which IIV4 is indicated $(32.0 \%$ aged 6 months-17 years, $33.8 \% \quad 18-65$ years, and $34.2 \%$ over 65 years). Pediatric subjects less than 9 years old $(n=139)$ received two doses. Following dose 1 and dose 2, 98.2\% and 100\%, respectively, returned the completed AERC recording any AEs. Following dose 1 and dose 2, 454 and 34 subjects, respectively, reported at least one $\mathrm{AE}$ (most frequently expected general and injection site symptoms and respiratory symptoms).

Conclusion: All reported AEs were expected as per summary product characteristics (smPC). No safety signals that impact public health or alter the benefit-risk profile of GSK's IIV4 were identified. Subjects from all vaccinated age groups were enrolled and the use of AERCs allowed rapid monitoring and analysis of reported AEs.

Trial Registration: ClinicalTrials.gov identifier, NCT03688620.

Funding: GlaxoSmithKline Biologicals SA.

Keywords: AlphaRix Tetra; Infectious disease; Influenza; Influsplit Tetra; Fluarix Tetra; Post marketing surveillance; Safety; Vaccination 


\section{INTRODUCTION}

The burden of seasonal influenza in Europe is substantial. A study published by the European Center for Disease Prevention and Control (ECDC) in 2018 ranked influenza as having the highest burden among 31 infectious diseases assessed. Influenza incidence and mortality were high with 5887 cases and 5.89 deaths per 100,000 population. Compared to the other infectious diseases, the burden of influenza ranked second for the age group under 15 years, third among adults (16-64 years), and was by far the highest among older people (over 65 years) [1].

Seasonal epidemics of influenza are caused by circulating influenza A virus subtypes and B virus lineages. Annual vaccination is the most effective approach to preventing influenza and its complications [2]. The influenza virus, however, undergoes frequent genetic and antigenic changes; therefore, each year new vaccines are formulated to match the circulating virus strains, based on recommendations from the World Health Organization (WHO) [3].

GSK's seasonal inactivated quadrivalent influenza vaccine (IIV4) is indicated for active immunization of adults and children from 6 months of age for the prevention of influenza disease caused by the two influenza $A$ virus subtypes and the two influenza B virus lineages contained in the vaccine.

As seasonal vaccines are typically reformulated annually and introduced in a short time span and to a large number of people, their benefit-risk profile must be routinely monitored and their safety rapidly assessed [4]. The European Medicines Agency (EMA) updated their guidance to vaccine manufacturers in this respect in 2014, no longer recommending small clinical trials for newly formulated vaccines prior to introduction, as these provided limited information on expected efficacy and safety [5]. Instead, they recommend early annual enhanced safety surveillance in one or several European Union member states, where data and systems permit the objective to be met quickly. The main objective is to rapidly detect and act on any new safety signals, i.e., increased frequency or severity of expected reactogenicity (e.g., local, systemic, or allergic events) intrinsic to each vaccine, compared to previous season or clinical trial rates, that could indicate a more serious risk [4].

Following an initial pilot study in the United Kingdom (UK) [6] in influenza season 2015/16, GSK refined an enhanced safety surveillance (ESS) methodology, using customized adverse event reporting cards (AERC). This was then successfully applied in the UK to assess vaccine safety in influenza seasons 2016/17 [7, 8] and 2017/18 (NCT03278067), mostly in adults and older adults because of preferential local recommendations for a live attenuated influenza vaccine (LAIV), except when contraindicated. In both studies, using the AERC increased reporting of EMA-specified adverse events of interest (AEIs) compared with electronic health records which require a medical visit.

The objective of the current ESS study for influenza season 2018/19 was to collect safety data for GSK's IIV4 in Belgium (AlphaRix Tetra), Germany (Influsplit Tetra), and Spain (Fluarix Tetra), for all age groups for which it is indicated. Of note, the different brand names by country refer to the same medicinal product (IIV4). The primary objective was to estimate the cumulative percentage of subjects reporting AEIs and/or other AEs, using the AERC, within 7 days following vaccination with IIV4. The secondary objectives were to estimate the weekly and cumulative data by age strata (6 months-17 years; 18-65 years; > 65 years), and risk status (at risk/not at risk). Results of the interim analysis showing weekly AEs reported from week 40 to 52 overall and by country are presented here.

\section{METHODS}

\section{Study Setting and Population}

This ESS study was a prospective multicenter study in Belgium, Germany, and Spain of GSK's IIV4 during the 2018/19 influenza season. Nine healthcare professionals (HCP) (three in each country) aimed to recruit a total of 1000 subjects (aged 18 years or older in Belgium and 
Germany, and 6 months or older in Spain) who received IIV4. Vaccination must have been performed in routine clinical practice according to local prescribing information, with one dose for subjects previously vaccinated against influenza or aged over 9 years, and two doses (4 weeks apart) for subjects aged under 9 years and not previously vaccinated against influenza [9]. Written informed consent/assent was obtained from subjects or their parents/guardians prior to enrollment. Subjects aged 6 months or older at the time of vaccination with IIV4, who consented and who would, according to investigators, comply with the protocol, were included in the study. Children in care were excluded from the study (i.e., children under the control or protection of an agency, organization, institution, or entity by the courts, the government, or a government body, acting in accordance with powers conferred on them by law or regulation).

The study was conducted between October 1 , 2018 and January 4, 2019 with recruitment planned from October 1 to December 31, 2018 for subjects requiring one dose, and from October 1 to December 1, 2018 for subjects requiring two doses. Study follow-up was approximately 8 days for subjects vaccinated with one dose and 36 days for subjects vaccinated with two doses.

\section{Selection of Countries}

A feasibility assessment of suitable countries for the conduct of this study was made and suitable research groups and sites were contacted. The study was conducted in Belgium, Germany, and Spain. Despite similar vaccine recommendations in these three countries (primarily targeting older adults and children at risk for annual influenza vaccination), sites in Spain that responded favorably were essentially pediatric sites, while Belgium and Germany have limited vaccine coverage in children. As such, Belgium and Germany only recruited subjects 18 years of age and above (with German participating sites targeting subjects aged 50 years and older). Spain targeted the enrollment of subjects between 6 months and 65 years of age at the time of the vaccination. This study was thus able to capture data on AEs in all age groups with an indication for GSK's seasonal influenza vaccine, regardless of the target population for vaccination.

\section{Safety Data Collection and Analysis}

This study aimed to capture AEs in near real time in order to rapidly estimate the reporting rate of AEIs experienced within days of vaccination. Weekly data reviews and four interim analyses were conducted. The results of the fourth interim analysis are presented here. An $\mathrm{AE}$ was defined as any untoward medical occurrence, temporally associated with the use of a medicinal product (whether or not related to the product) or with a study procedure. The HCPs provided each vaccinee or their parent/guardian with a customized AERC to be completed from the day of vaccination and for the following 6 days. The AERC includes predefined categories of AEIs representing events commonly associated with IIV4 s, along with a free text field to report any other $\mathrm{AE}$, and the possibility to indicate that no $\mathrm{AE}$ occurred in the 7-day timeframe. Subjects were asked to return the AERC to their HCP at the next scheduled visit or by mail within 2 weeks of vaccination. Practice staff then entered the information in an electronic Case Report Form (eCRF), using a code list provided to each practice to standardize data collection and analysis of AEIs. Details on the AERC and eCRF were previously published by the authors [10]. Reported AEIs were coded using the Medical Dictionary for Regulatory Activities (MedDRA) classification [11].

\section{Statistical Considerations}

In the sample size calculations, the probability of observing at least one $\mathrm{AE}$ was presented according to several scenarios in which the expected number of vaccinees and true percentage of subjects experiencing an $\mathrm{AE}$ were tabulated. Those scenarios were previously reported in the published protocol [10]. Of note, the planned number of study subjects to 
be enrolled ( $n=1000)$ was expected to enable detection of the very common $(\geq 1 / 10)$, common $(\geq 1 / 100$ to $<1 / 10)$, uncommon $(\geq 1 /$ 1000 to $<1 / 100)$ AEIs, and/or other AEs.

Demographic data collected for all subjects, including age, gender, geographic ancestry, and risk status, are presented for the overall study population. The HCP assessed and recorded each subject as at risk/not at risk for influenzaassociated morbidity and mortality according to the local recommendations. Of note, in Europe, according to ECDC [12], risk groups should include pregnant women, subjects with chronic conditions (e.g., heart or lung diseases, metabolic or renal disease, chronic liver disease, chronic neurological conditions, or immunodeficiencies), older subjects in long-term care facilities, children aged 6-59 months, and healthcare workers. The interim analysis was conducted on all vaccinated subjects who received the AERC. The number and percentage of vaccinated subjects are presented by country and center, by International Standards Organization (ISO) week) of vaccination, and by age category (6 months-17 years, $18-65$ years, over 65 years). The number and percentage of subjects returning their AERC are presented by country/center and for dose 1 and dose 2 . The occurrences of AEs are presented by AEI or other AE by Preferred Term (PT) by vaccination week (expected ISO weeks 40-52) using the MedDRA classification for Primary System Organ Class, overall and by country. All analyses were descriptive in nature and did not aim to allow direct comparisons. The extended Clopper-Pearson exact confidence interval (CI) for cluster data was used to compute $95 \%$ CI on all estimated percentages, accounting for clustering effect of centers [13].

\section{Compliance with Ethics Guidelines}

The study was conducted in accordance with the International Conference on Harmonisation (ICH) Guideline for Good Clinical Practice, Good Epidemiology Practice, Good Pharmacovigilance Practice, or other applicable guidelines, all applicable subject privacy requirements and the guiding principles of the
Declaration of Helsinki. The study was designed and conducted in accordance with the ICH Harmonised Tripartite Guideline for clinical investigation of medicinal products in the paediatric population (ICH E11) and all other applicable ethical guidelines. GSK obtained favourable opinion/approval to conduct the study prior to a site initiating the study in that country or has documented that neither a favourable opinion nor an approval to conduct the study was needed. Written informed consent/assent was obtained from subjects or their parents/guardians prior to enrolment. The study received approval from the following ethics committees (ECs), or institutional review boards (IRBs) and regional authorities were consulted consistent with country requirements, namely Commissie Medische Ethiek UZ/ KU Leuven (EC) in Belgium (approval received 24 August); Ethik-Kommission der Bayerischen (EC) in Germany (approval received 20 September), and in Spain the PEIBA (IRB and RA combined) for Andalusia (approval received 26 September) and Hospital Vall de Hebron (IRB) and Goli Gurina (RA) for Catalonia (approval received 22 October). GSK posted the study protocol on GSK's Clinical Study Register (accessible at www.gsk-clinicalstudyregister.com, GSK Study ID 207737) and on the US National Library of Medicine website www.clinicaltrials. gov (NCT03688620).

\section{RESULTS}

In total, 1060 vaccinated subjects were included between October 5, 2018 (ISO week 40) and December 31, 2018 (ISO week 52), of which 329 (31.0\%) were from Belgium, 278 (26.2\%) were from Germany, and $453(42.7 \%)$ were from Spain (Table 1). All subjects were vaccinated with dose 1 and 139 subjects aged less than 9 years in Spain received dose 2 . All subjects in the age category 6 months -17 years $(n=339$, $32.0 \%)$ were from Spain, while 358 subjects (33.8\%) aged 18-65 years were from all three countries and 363 subjects (34.2\%) aged over 65 years were from Belgium and Germany (Fig. 1). The median age of subjects was 68 years in Belgium and Germany and 6 years in Spain 
Table 1 Number of vaccinated subjects enrolled by center and by age group

\begin{tabular}{|c|c|c|c|c|c|c|}
\hline \multirow[t]{2}{*}{ Country } & \multicolumn{6}{|c|}{ Total vaccinated subjects enrolled, $N=1060$} \\
\hline & Center & $N$ & $\%^{\mathrm{a}}$ & Age group & $N$ & $\%^{a}$ \\
\hline \multirow[t]{4}{*}{ Belgium } & 237303 & 129 & 12.2 & 6 months -17 years & 0 & 0 \\
\hline & 237304 & 36 & 3.4 & $18-65$ years & 133 & 12.5 \\
\hline & 237305 & 164 & 15.5 & $>65$ years & 196 & 18.5 \\
\hline & All & 329 & 31.0 & All & 329 & 31.0 \\
\hline \multirow[t]{4}{*}{ Germany } & 237278 & 135 & 12.7 & 6 months -17 years & 0 & 0 \\
\hline & 237279 & 93 & 8.8 & $18-65$ years & 111 & 10.5 \\
\hline & 237280 & 50 & 4.7 & $>65$ years & 167 & 15.8 \\
\hline & All & 278 & 26.2 & All & 278 & 26.2 \\
\hline \multirow[t]{4}{*}{ Spain } & 236682 & 250 & 23.6 & 6 months -17 years & 339 & 32.0 \\
\hline & 236684 & 153 & 14.4 & $18-65$ years & 114 & 10.8 \\
\hline & 237680 & 50 & 4.7 & $>65$ years & 0 & 0 \\
\hline & All & 453 & 42.7 & All & 453 & 42.7 \\
\hline
\end{tabular}

$N$ total number of subjects, $n$ number and \% (percentage) of subjects in a given center or age group and country

a Due to the rounding of the percentages to 1 decimal, the total may not equal $100 \%$

(mean age overall 42.8 years). Overall $51.3 \%$ of subjects were female and $98.0 \%$ were Caucasian. Subjects assessed as being at risk for influenzaassociated morbidity and mortality were distributed as follows: 204 (62.0\%) in Belgium, 133 (47.8\%) in Germany, and 223 (49.2\%) in Spain (Table 2). For those subjects receiving two doses, median age was 3 years, $42.2 \%$ were female, 99.3\% were Caucasian, and $34.5 \%$ were assessed as at risk for influenza-associated morbidity and mortality.

The majority of subjects aged 6 months-17 years received dose 1 in ISO weeks 42-45 in Spain. Most adults (18-65 years) in Belgium and Germany were vaccinated earlier (in ISO week 42 for Belgium and ISO weeks 40-42 for Germany) while those in Spain were mostly vaccinated later (ISO weeks 45-46). Among the older adults ( $>65$ years), most in Germany were vaccinated early (ISO weeks 40-42) while those in Belgium were typically vaccinated later (ISO weeks 43-46) (Fig. 1).

Following each vaccination, all subjects received an AERC to complete and return.
Overall, by January $14,2019,98.2 \%$ of AERCs were returned $(94.8 \%$ in Belgium, $99.6 \%$ in Germany, and $99.8 \%$ in Spain) for dose 1 . For dose 2 in Spain, 100\% of AERCs were returned (Table 3).

The percentage of subjects reporting AEIs and other AEs (classed according to MedDRA Primary System Organ Class [PSOC] and Preferred Term [PT]) is presented for all countries following dose 1 (Table 4) and dose 2 (Table 5).

Overall, among the 1060 vaccinated subjects, 454 subjects $(42.8 \%)$ reported at least one $\mathrm{AE}$ after dose 1 . The most frequently reported AEs were on the predefined list of AEIs, i.e., injection site pain (17.5\%), rhinorrhea (8.6\%), injection site swelling (7.5\%), headache (7.4\%), myalgia $(6.7 \%)$, cough $(6.7 \%)$, fatigue $(6.5 \%)$, injection site erythema (5.3\%), nasal congestion $(5.3 \%)$, and oropharyngeal pain $(4.6 \%)$. Some predefined AEIs were not reported, i.e., there were no cases of anaphylaxis, generalized rash, febrile convulsion, Bell's palsy, or Guillain-Barré syndrome. Other AEIs were less frequent, reported in $0.2-3.4 \%$ of subjects. 


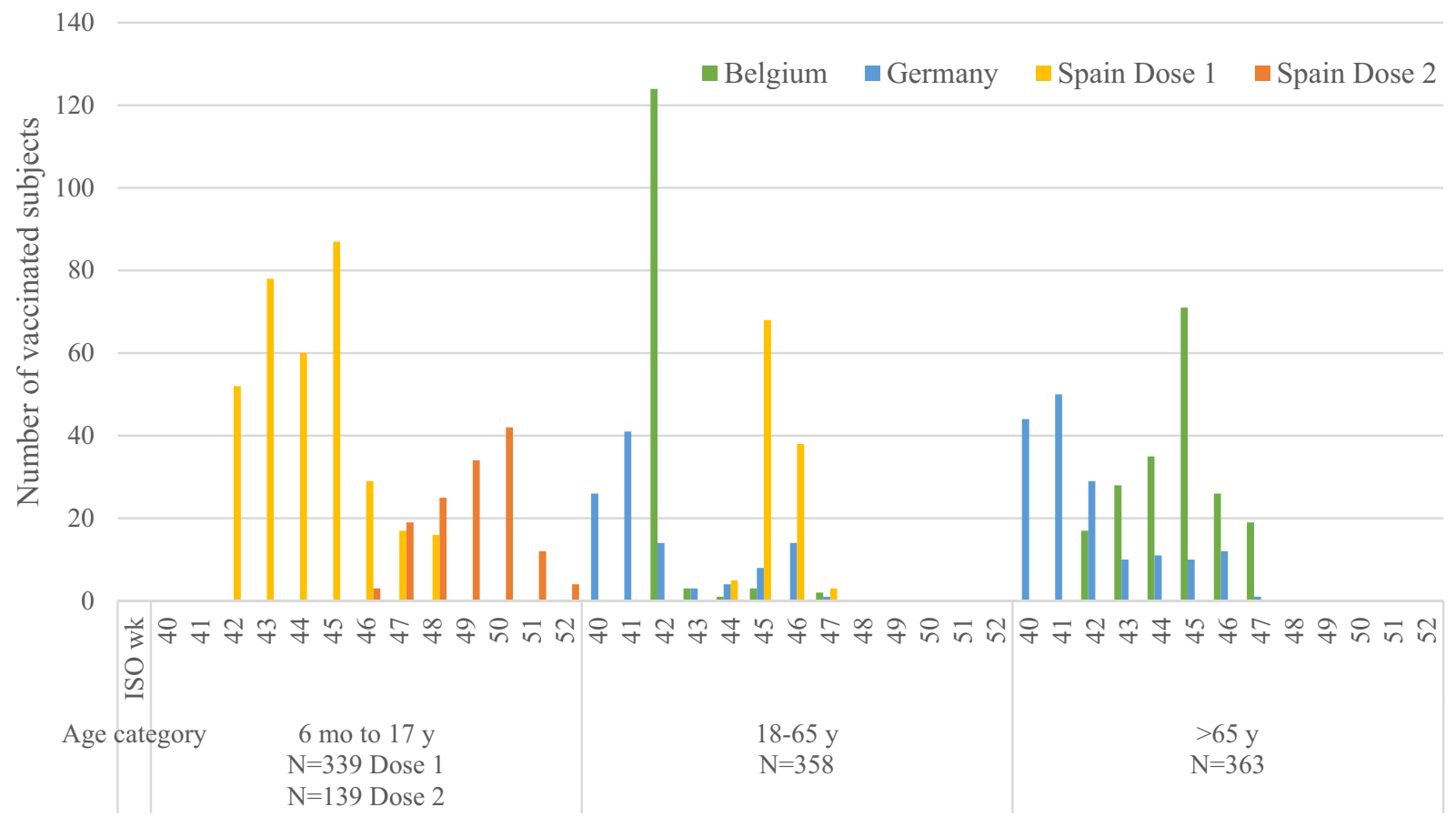

Fig. 1 Number of subjects vaccinated during the study using dose 1 and dose 2, by age category (pediatric, 6 months -17 years; adult, 18-65 years; older adult, $>65$ years), country, and per ISO week (40-52).

Regarding AEs not on the predefined list, the most frequently reported was pruritus $(0.8 \%)$, while other AEs were reported for $0.1-0.4 \%$ of subjects. The most frequently reported MedDRA PSOC of AEs were general disorders and administration site conditions ( $n=301,28.4 \%$ ), followed by respiratory, thoracic , and mediastinal disorders $(n=184,17.4 \%)$, musculoskeletal and connective tissue disorders $(n=95,9.0 \%)$, nervous system disorders (including headache) $(n=85,8.0 \%)$, gastrointestinal disorders $(n=67,6.3 \%)$, and infections and infestations $(n=66,6.2 \%)$. The remaining classes of AEs were reported by $3 \%$ or fewer subjects (Table 4).

The overall frequency of AEs reported after dose 1 was similar (i.e., $41.9 \%$ in Belgium, $36.7 \%$ in Germany, and $47.2 \%$ in Spain), and the ranking and frequencies of the most common classes of AEs were mostly of similar magnitude: general disorders reported by $26.7-30.0 \%$ of subjects, respiratory disorders by 11.5-23.0\%, musculoskeletal disorders by
Two colors are used for Spain, where some pediatric subjects received two vaccination doses. ISO wk International Standards Organization week, mo month, $N$ total number of subjects in category, y year

8.3-10.3\%, nervous system disorders (including headache) by $7.9-8.2 \%$, gastrointestinal disorders by $5.0-6.8 \%$, and infections by $4.6-7.9 \%$. In Spain, metabolism disorders (i.e., decreased appetite) were also reported by $5.3 \%$ of subjects.

In Spain, among the 139 pediatric subjects who received dose 2,34 subjects reported at least one $\mathrm{AE}$. The most frequently reported AEs were on the predefined list of AEIs and were classed as respiratory, thoracic, and mediastinal disorders $(n=21,15.1 \%)$ of which cough $(8.6 \%)$, rhinorrhea $(6.5 \%)$, and nasal congestion (5.0\%) were most frequent. The next most frequently reported AEIs were general disorders and administration site conditions $(n=11$, $7.9 \%)$ of which injection site pain $(5.0 \%)$ and pyrexia $(4.3 \%)$ were most frequent. Other AEIs were reported by $0.7-2.9 \%$ of subjects. There were no reports of the following AEIs: headache, febrile convulsion, anaphylaxis, hypersensitivity, generalized rash, arthropathy, nausea, injection site swelling or erythema, fatigue, chills, face edema, oropharyngeal pain, Bell's 
Table 2 Summary of demographic characteristics and risk status for influenza-associated morbidity and mortality (ISO weeks $40-52$, dose 1 )

\begin{tabular}{|c|c|c|c|c|c|c|c|c|}
\hline & \multicolumn{2}{|c|}{ Belgium $N=329$} & \multicolumn{2}{|c|}{ Germany $N=278$} & \multicolumn{2}{|c|}{ Spain $N=453$} & \multicolumn{2}{|c|}{ Overall $N=1060$} \\
\hline & $\begin{array}{l}\text { Value } \\
\text { or } n\end{array}$ & $\%$ & $\begin{array}{l}\text { Value } \\
\text { or } n\end{array}$ & $\%$ & $\begin{array}{l}\text { Value } \\
\text { or } n\end{array}$ & $\%$ & $\begin{array}{l}\text { Value } \\
\text { or } n\end{array}$ & $\%$ \\
\hline \multicolumn{9}{|l|}{ Age (year) at dose 1 vaccination } \\
\hline Median & 68.0 & & 68.5 & & 6.0 & & 48.0 & \\
\hline Maximum & 91 & & 100 & & 64 & & 100 & \\
\hline $\begin{array}{l}\text { Minimum age (month/year) } \\
\text { at dose } 1 \text { vaccination }\end{array}$ & 23 years & & 18 years & & 6 months & & 6 months & \\
\hline \multicolumn{9}{|l|}{ Age category at dose 1 vaccination } \\
\hline 6 months -17 years & 0 & 0.0 & 0 & 0.0 & 339 & 74.8 & 339 & 32.0 \\
\hline $18-65$ years & 133 & 40.4 & 111 & 39.9 & 114 & 25.2 & 358 & 33.8 \\
\hline$>65$ years & 196 & 59.6 & 167 & 60.1 & 0 & 0.0 & 363 & 34.2 \\
\hline \multicolumn{9}{|l|}{ Gender } \\
\hline Female & 147 & 44.7 & 166 & 59.7 & 231 & 51.0 & 544 & 51.3 \\
\hline Male & 182 & 55.3 & 112 & 40.3 & 222 & 49.0 & 516 & 48.7 \\
\hline \multicolumn{9}{|l|}{ Geographic ancestry } \\
\hline Black or African American & 0 & 0.0 & 2 & 0.7 & 1 & 0.2 & 3 & 0.3 \\
\hline Asian-Central/South Asian & 1 & 0.3 & 1 & 0.4 & 1 & 0.2 & 3 & 0.3 \\
\hline Asian-East Asian & 4 & 1.2 & 1 & 0.4 & 0 & 0.0 & 5 & 0.5 \\
\hline Asian-South East Asian & 2 & 0.6 & 0 & 0.0 & 0 & 0.0 & 2 & 0.2 \\
\hline White-Arabic/North African & 1 & 0.3 & 0 & 0.0 & 3 & 0.7 & 4 & 0.4 \\
\hline White-Caucasian/European & 321 & 97.6 & 274 & 98.6 & 444 & 98.0 & 1039 & 98.0 \\
\hline Other & 0 & 0.0 & 0 & 0.0 & 4 & 0.9 & 4 & 0.4 \\
\hline \multicolumn{9}{|c|}{ Risk status: influenza-associated morbidity and mortality ${ }^{a}$} \\
\hline At risk & 204 & 62.0 & 133 & 47.8 & 223 & 49.2 & 560 & 52.8 \\
\hline Not at risk & 125 & 38.0 & 145 & 52.2 & 230 & 50.8 & 500 & 47.2 \\
\hline
\end{tabular}

$I S O$ International Standards Organization, $N$ number of subjects vaccinated, $n$ number and \% (percentage) of vaccinated subjects in a given category

${ }^{\text {a }}$ Assessed by the HCP based on his/her judgment and experience

palsy, or Guillain-Barré syndrome. AEs not on the predefined list were reported by $0.7-1.4 \%$ of subjects (i.e., one case each of sneezing and abdominal pain and two cases of eye pruritus) (Table 5).

In addition, during the study period, according to HCPs judgement, no serious AEs 
Table 3 Number (\%) of subjects who returned the AERC by center and country

\begin{tabular}{|c|c|c|c|c|c|c|c|}
\hline \multirow[t]{3}{*}{ Country } & \multirow[t]{3}{*}{ Center } & \multicolumn{3}{|c|}{ Dose 1} & \multicolumn{3}{|c|}{ Dose 2} \\
\hline & & \multirow[t]{2}{*}{$N$} & \multicolumn{2}{|c|}{ AERC returned } & \multirow[t]{2}{*}{$N$} & \multicolumn{2}{|c|}{ AERC returned } \\
\hline & & & $n$ & $\%$ & & $\bar{n}$ & $\%$ \\
\hline \multirow[t]{4}{*}{ Belgium } & 237303 & 129 & 112 & 86.8 & - & - & - \\
\hline & 237304 & 36 & 36 & 100 & - & - & - \\
\hline & 237305 & 164 & 164 & 100 & - & - & - \\
\hline & All & 329 & 312 & 94.8 & - & - & - \\
\hline \multirow[t]{4}{*}{ Germany } & 237278 & 135 & 134 & 99.3 & - & - & - \\
\hline & 237279 & 93 & 93 & 100 & - & - & - \\
\hline & 237280 & 50 & 50 & 100 & - & - & - \\
\hline & All & 278 & 277 & 99.6 & - & - & - \\
\hline \multirow[t]{4}{*}{ Spain } & 236682 & 250 & 250 & 100 & 116 & 116 & 100 \\
\hline & 236684 & 153 & 152 & 99.3 & 4 & 4 & 100 \\
\hline & 237680 & 50 & 50 & 100 & 19 & 19 & 100 \\
\hline & All & 453 & 452 & 99.8 & 139 & 139 & 100 \\
\hline Overall & All & 1060 & 1041 & 98.2 & 139 & 139 & 100 \\
\hline
\end{tabular}

$A E R C$ adverse event reporting card, $N$ number of subjects vaccinated who received the AERC, $n$ number and \% (percentage) of subjects vaccinated who returned the AERC as specified

deemed to be related to GSK's IIV4 were reported within 7 days post vaccination.

In the electronic supplementary material, the percentage of subjects with AEs by ISO week following dose 1 and dose 2 is presented overall and by country. See Tables S1-S5 in the electronic supplementary material for details.

\section{DISCUSSION}

At the interim analysis in this study, $100 \%$ of AERCs were returned for two out of three centers in each country, while the return rate was $86.8-99.3 \%$ for the remaining centers for dose 1 . For dose $2,100 \%$ of AERCs were returned. Following dose 1, interim results showed that 454 subjects reported at least one $\mathrm{AE}$ within 7 days of vaccination among the 1060 subjects. The most frequently reported were on the predefined list of AEIs, i.e., local injection site reactions and general symptoms (headache, fatigue), respiratory symptoms (rhinorrhea, cough, nasal congestion, oropharyngeal pain), and myalgia. These AEIs were expected events commonly observed after vaccination with an IIV4 [14].

Ongoing reviews of raw data during the study period and the interim analyses did not detect any safety signals that could affect public health or could alter the benefit-risk profile of GSK's IIV4.

The methodology used for data collection and analysis (i.e., customized AERC completed by subjects and coded into eCRFs by practice staff using a MedDRA-based coding list) also appears to have been successful and a promising approach, with a return rate for AERCs that was in the same range or above the return rate observed in active surveillance. There are two possible explanations for the high return rate: firstly, feedback following similar studies in the 
Table 4 Number (\%) of subjects reporting AEIs and other AEs within 7 days post-dose 1 (i.e., vaccination day and following 6 days) using AERC for all countries $(N=1060)$

\begin{tabular}{|c|c|c|}
\hline \multirow{2}{*}{$\begin{array}{l}\text { MedDRA primary system organ class (CODE) } \\
\text { Preferred term (code) }\end{array}$} & \multicolumn{2}{|c|}{$N=1060$} \\
\hline & $\bar{n}$ & $\%$ \\
\hline Any & 454 & 42.8 \\
\hline Any general disorders and administration site conditions (10018065) & 301 & 28.4 \\
\hline Injection site pain $(10022086)^{*}$ & 185 & 17.5 \\
\hline Injection site swelling $(10053425)^{*}$ & 80 & 7.5 \\
\hline Fatigue $(10016256)^{*}$ & 69 & 6.5 \\
\hline Injection site erythema $(10022061)^{*}$ & 56 & 5.3 \\
\hline Pyrexia $(10037660)^{*}$ & 33 & 3.1 \\
\hline Chills $(10008531)^{*}$ & 25 & 2.4 \\
\hline Injection site pruritus (10022093) & 4 & 0.4 \\
\hline Face edema $(10016029)^{*}$ & 2 & 0.2 \\
\hline $\begin{array}{l}\text { Influenza-like illness (10022004); mucous membrane } \\
\text { disorder (10028133); peripheral swelling (10048959) }\end{array}$ & 2 each & 0.2 each \\
\hline $\begin{array}{l}\text { Discomfort }(10013082) \text {; feeling cold }(10016326) \text {; feeling hot } \\
(10016334) \text {; injection site hematoma }(10022066) \text {; injection site } \\
\text { reaction }(10022095) \text {; injection site warmth }(10022112) \text {; } \\
\text { malaise (10025482) }\end{array}$ & 1 each & 0.1 each \\
\hline Any respiratory, thoracic, and mediastinal disorders (10038738) & 184 & 17.4 \\
\hline Rhinorrhea $(10039101)^{*}$ & 91 & 8.6 \\
\hline Cough $(10011224)^{*}$ & 71 & 6.7 \\
\hline Nasal congestion $(10028735)^{*}$ & 56 & 5.3 \\
\hline Oropharyngeal pain $(10068319)^{*}$ & 49 & 4.6 \\
\hline Dysphonia $(10013952)^{*}$ & 23 & 2.2 \\
\hline Epistaxis $(10015090)^{*}$ & 9 & 0.8 \\
\hline Wheezing $(10047924)^{*}$ & 6 & 0.6 \\
\hline Dyspnea (10013968); sneezing (10041232) & 3 each & 0.3 each \\
\hline $\begin{array}{l}\text { Dry throat }(10013789) \text {; increased upper airway secretion }(10062717) \text {; } \\
\text { pharyngeal inflammation }(10065716) ; \text { throat clearing }(10080125)\end{array}$ & 1 each & 0.1 each \\
\hline Any musculoskeletal and connective tissue disorders (10028395) & 95 & 9.0 \\
\hline Myalgia $(10028411)^{*}$ & 71 & 6.7 \\
\hline Arthropathy $(10003285)^{*}$ & 32 & 3.0 \\
\hline Pain in extremity (10033425) & 3 & 0.3 \\
\hline Rheumatic disorder (10072736) & 2 & 0.2 \\
\hline
\end{tabular}


Table 4 continued

\begin{tabular}{lll}
\hline MedDRA primary system organ class (CODE) & \multicolumn{1}{l}{$\mathbf{1 0 6 0}$} & $\mathbf{\%}$ \\
\cline { 2 - 3 } Preferred term (code) & $\boldsymbol{n}$ & 0.1 each \\
\hline Limb discomfort (10061224); muscle spasms (10028334); & 1 each & 8.0 \\
musculoskeletal pain (10028391); synovial cyst (10042858) & & 7.4 \\
Any nervous system disorders (10029205) & 85 & 0.4 \\
Headache (10019211)* & 78 & 0.1 each \\
Dizziness (10013573) & 4 & \\
Aphonia (10002953); poor quality sleep (10062519); & 1 each \\
somnolence (10041349); tremor (10044565) & & 0.0 \\
Febrile convulsion (10016284)* & 0 & 6.3 \\
Any gastrointestinal disorders (10017947) & 67 & 3.0 \\
Diarrhea (10012735)* & 32 & 3.0 \\
Nausea (10028813)* & 32 & 1.8 \\
Vomiting (10047700)* & 19 & 0.3 \\
Abdominal pain (10000081) & 3 & 0.1 each \\
Gingival bleeding (10018276); lip swelling (10024570); & 1 each \\
stomatitis (10042128); tongue discomfort (10077855) & & 6.2 \\
Any infections and infestations (10021881) & 66 & 3.4 \\
Conjunctivitis (10010741)* & 36 & 2.8 \\
Rhinitis (10039083)* & 30 & 0.3 \\
Nasopharyngitis (10028810) & 3 & 0.1 each \\
Bronchitis (10006451); ear infection (10014011); herpes simplex & &
\end{tabular}

(10019948); hordeolum (10020377); laryngitis (10023874);

oral herpes (10067152); upper respiratory tract infection (10046306)

Any metabolism and nutrition disorders (10027433)

Decreased appetite (10061428)*

32

3.0

Any psychiatric disorders (10037175)

$19 \quad 1.8$

Irritability (10022998)*

Insomnia (10022437); mood swings (10027951);

1 each

0.1 each

sleep disorder (10040984)

Any skin and subcutaneous tissue disorders (10040785)

$\begin{array}{ll}17 & 1.6 \\ 8 & 0.8 \\ 7 & 0.7 \\ 2 & 0.2 \\ 1 \text { each } & 0.1 \text { each }\end{array}$

Pruritus (10037087)

0.1 each 
Table 4 continued

\begin{tabular}{lll}
\hline MedDRA primary system organ class (CODE) & $\boldsymbol{N = \mathbf { 1 0 6 0 }}$ & $\mathbf{\%}$ \\
\cline { 2 - 3 } Preferred term (code) & $n$ & 0.0 \\
\hline Rash generalized (10037858)* & 0 & 0.7 \\
Any ear and labyrinth disorders (10013993) & 7 & 0.2 each \\
Ear pain (10014020); vertigo (10047340) & 2 each & 0.1 each \\
Ear hemorrhage (10014009); ear pruritus (10052138); & 1 each & 0.6 \\
tinnitus (10043882) & & 0.6 \\
Any immune system disorders (10021428) & 6 & 0.0 \\
Hypersensitivity (10020751)* & 6 & 0.4 \\
Anaphylactic reaction (10002198)* & 0 & 0.4 \\
Any investigations (10022891) & 4 & 0.3 \\
Heart rate increased (10019303) & 4 & 0.1 each \\
Any eye disorders (10015919) & 3 & 0.1 \\
Eye pruritus (10052140); lacrimation increased (10023644); & 1 each \\
swelling of eyelid (10042690) & & 0.1 \\
Any injury, poisoning, and procedural complications (10022117) & 1 & 0.1 \\
Muscle strain (10050031) & 1 & 0.1 \\
Any cardiac disorders (10007541) & 1 & 1 \\
Tachycardia (10043071) & 1 & \\
\hline
\end{tabular}

$A E$ adverse event, $A E I$ adverse event of interest, AERC adverse event reporting card, MedDRA Medical Dictionary for Regulatory Activities [11], $N$ total number of subjects, $n$ number of subjects in category

*AEI

UK has revealed a growing interest from patients to participate actively in their health follow-up; and secondly, the study included a planned visit following vaccination, to encourage continued subject participation (see protocol [10]). In Italy, active surveillance 7 days after influenza vaccination involving a follow-up telephone interview or web-based platform resulted in a 97\% response rate overall [15]. In the Netherlands, a web-based questionnaire had response rates of $90.7 \%$ at day $5,84.5 \%$ at day 15 , and $78.4 \%$ at day 30 after influenza vaccination [16].

This ESS of GSK's IIV4 covered all age groups for which the vaccine is indicated. This was achieved through a combination of data from three European countries: Spain, where pediatric subjects were enrolled (from 6 months of age), as well as Germany and Belgium, all of which use IIV4 in adults and older adults. The distribution of subjects across countries and across age categories was generally balanced. The target number of patients was reached in the 3-month recruitment period, with sufficient data collected in a relatively short period of time, as the majority of subjects were vaccinated and enrolled within a 4-week timeframe. Data were also reviewed on an ongoing basis in near real time owing to the electronic application and data collection methods used.

A recent review summarizes several other initiatives conducted so far to address the EMA requirement for enhanced safety surveillance of seasonal influenza vaccines, and illustrates a 
Table 5 Number (\%) of subjects reporting AEIs and other AEs within 7 days post-dose 2 (i.e., vaccination day and following 6 days) using AERC in Spain

\begin{tabular}{|c|c|c|}
\hline \multirow{2}{*}{$\begin{array}{l}\text { MedDRA primary system organ class (CODE) } \\
\text { Preferred term (code) }\end{array}$} & \multicolumn{2}{|c|}{$N=139$} \\
\hline & $\bar{n}$ & $\%$ \\
\hline Any & 34 & 24.5 \\
\hline Any respiratory, thoracic, and mediastinal disorders (10038738) & 21 & 15.1 \\
\hline Cough $(10011224)^{*}$ & 12 & 8.6 \\
\hline Rhinorrhea $(10039101)^{*}$ & 9 & 6.5 \\
\hline Nasal congestion $(10028735)^{*}$ & 7 & 5.0 \\
\hline Dysphonia $(10013952)^{*}$ & 3 & 2.2 \\
\hline Wheezing $(10047924)^{*}$ & 2 & 1.4 \\
\hline Epistaxis $(10015090)^{*}$ & 1 & 0.7 \\
\hline Sneezing (10041232) & 1 & 0.7 \\
\hline Oropharyngeal pain $(10068319)^{*}$ & 0 & 0.0 \\
\hline Any general disorders and administration site conditions (10018065) & 11 & 7.9 \\
\hline Injection site pain $(10022086)^{*}$ & 7 & 5.0 \\
\hline Pyrexia $(10037660)^{*}$ & 6 & 4.3 \\
\hline Chills $(10008531)^{*}$ & 0 & 0.0 \\
\hline Face edema $(10016029)^{*}$ & 0 & 0.0 \\
\hline Fatigue $(10016256)^{*}$ & 0 & 0.0 \\
\hline Injection site erythema $(10022061)^{*}$ & 0 & 0.0 \\
\hline Injection site swelling $(10053425)^{*}$ & 0 & 0.0 \\
\hline Any infections and infestations (10021881) & 5 & 3.6 \\
\hline Conjunctivitis $(10010741)^{*}$ & 4 & 2.9 \\
\hline Rhinitis $(10039083)^{*}$ & 1 & 0.7 \\
\hline Any gastrointestinal disorders (10017947) & 4 & 2.9 \\
\hline Diarrhea $(10012735)^{*}$ & 2 & 1.4 \\
\hline Vomiting $(10047700)^{*}$ & 1 & 0.7 \\
\hline Abdominal pain (10000081) & 1 & 0.7 \\
\hline Nausea $(10028813)^{*}$ & 0 & 0.0 \\
\hline Any metabolism and nutrition disorders (10027433) & 4 & 2.9 \\
\hline Decreased appetite $(10061428)^{*}$ & 4 & 2.9 \\
\hline Any psychiatric disorders (10037175) & 4 & 2.9 \\
\hline Irritability $(10022998)^{*}$ & 4 & 2.9 \\
\hline Any eye disorders (10015919) & 2 & 1.4 \\
\hline
\end{tabular}


Table 5 continued

\begin{tabular}{|c|c|c|}
\hline \multirow{2}{*}{$\begin{array}{l}\text { MedDRA primary system organ class (CODE) } \\
\text { Preferred term (code) }\end{array}$} & \multicolumn{2}{|c|}{$N=139$} \\
\hline & $n$ & $\%$ \\
\hline Eye pruritus (10052140) & 2 & 1.4 \\
\hline Any musculoskeletal and connective tissue disorders (10028395) & 1 & 0.7 \\
\hline Myalgia $(10028411)^{*}$ & 1 & 0.7 \\
\hline Arthropathy $(10003285)^{*}$ & 0 & 0.0 \\
\hline Any skin and subcutaneous tissue disorders (10040785) & 1 & 0.7 \\
\hline Rash $(10037844)^{*}$ & 1 & 0.7 \\
\hline Rash generalized $(10037858)^{*}$ & 0 & 0.0 \\
\hline Any immune system disorders (10021428) & 0 & 0.0 \\
\hline Anaphylactic reaction $(10002198)^{*}$ & 0 & 0.0 \\
\hline Hypersensitivity $(10020751)^{*}$ & 0 & 0.0 \\
\hline Any nervous system disorders (10029205) & 0 & 0.0 \\
\hline Febrile convulsion $(10016284)^{*}$ & 0 & 0.0 \\
\hline Headache $(10019211)^{*}$ & 0 & 0.0 \\
\hline
\end{tabular}

$A E$ adverse event, $A E I$ adverse event of interest, $A E R C$ adverse event reporting card, MedDRA Medical Dictionary for Regulatory Activities [11], $N$ total number of subjects, $n$ number of subjects in category

${ }^{*}$ AEI

diverse range of approaches used in Europe (e.g., patient diaries, patient records, interviews) with their challenges. Several studies reported difficulties with the recruitment of pediatric participants or with achieving high response rates [17].

A key strength of this study was the inclusion of a large and diverse group of subjects of all ages for which IIV4 is indicated, and from participating countries with different vaccination practices. Nevertheless, the relatively low number of subjects included did not allow investigation of potential disparities, across age groups notably, which deserves further attention in the future. The study procedures and prospective data collection proved to be successful, given the high return rate of AERCs. The use of an electronic application to collect standardized data (eCRFs using the coded AEI list) simplified data extraction, cleaning, and analysis to facilitate near-real-time assessment of the safety data, and will allow for future comparisons. The main limitations of this observational study were the absence of formal baseline rates for comparison (although final results can be discussed in light of the expected $\mathrm{AE}$ rates from clinical trials reported in the smPC), the limited timeframe for AE detection which is not adequate to fully capture AEs with a longer risk period, and that the enrollment period was dependent on the timing of vaccination campaigns in participating countries. Enrollment, thus, could not begin before mid to end October at most participating centers. This was, however, mitigated by a fast recruitment period, condensed into approximately 4 weeks. Given the small number of participating centers, the participants (mainly white-Caucasian) may not be representative of all inhabitants in these countries. Furthermore, the sample size was not large enough to allow for the assessment of rare AEs; however, on the basis of the EMA guidance, this was not an objective of the study. 


\section{CONCLUSION}

Importantly, this interim analysis did not show any safety signals for GSK's IIV4 in the 2018/19 influenza season that could impact public health or the known safety profile of the vaccine. Further analyses will describe the reported AEs in more detail and confirm the current safety findings. This multi-country study and the proposed ESS methodology were successful in rapidly providing safety data from subjects of all ages for whom IIV4 is indicated. It offered a suitable approach for the continuous monitoring of seasonal influenza vaccines, overcoming the underreporting frequently observed with passive initiatives.

\section{ACKNOWLEDGEMENTS}

The authors thank the participating patients, the medical staff who contributed to study implementation, and the clinical practices that allowed pseudonymised data to be extracted from their general practice CMR system. In addition, the authors thank the GSK's clinical operational teams for their continuous support who ensured a successful study conduct. The authors also thank Jacqueline Miller, Dominique Rosillon, Stephanie Gilon, Alexander Schmidt, Katie Smith, Elvira Zandman Van Dijk, Harish Dasari, Christiane Klein, Lode Godderis, Stephane Vanden Bemden, Hugo Loos, and Xavier Martinez Gomez for their contribution to this study/publication.

AlphaRix Tetra, Influsplit Tetra, and Fluarix Tetra are trademarks of the GSK group of companies.

Funding. GlaxoSmithKline Biologicals SA was the funding source and was involved in all stages of the study. GlaxoSmithKline Biologicals SA also funded the Rapid Service and Open Access Fees.

Medical Writing, Editorial and Other Assistance. The authors thank Business \& Decision Life Sciences platform for editorial assistance and manuscript coordination, on behalf of GSK. Kavi Littlewood provided writing support, and Bruno Dumont coordinated manuscript development and editorial support.

Authorship. All named authors meet the International Committee of Medical Journal Editors (ICMJE) criteria for authorship for this article, take responsibility for the integrity of the work as a whole, and have given their approval for this version to be published.

Disclosures. Vishvesh Shende declares he is employed by VPN Consultancy Limited working on behalf of the GSK group of companies. Anne Yeakey is employed by the GSK group of companies and hold shares in the GSK group of companies. Gael Dos Santos is employed by the GSK group of companies and hold shares in the GSK group of companies. Silvia Damaso is employed by the GSK group of companies and hold shares in the GSK group of companies.

Compliance with Ethics Guidelines. The study was conducted in accordance with the International Conference on Harmonisation (ICH) Guideline for Good Clinical Practice, Good Epidemiology Practice, Good Pharmacovigilance Practice, or other applicable guidelines, all applicable subject privacy requirements and the guiding principles of the Declaration of Helsinki. The study was designed and conducted in accordance with the ICH Harmonised Tripartite Guideline for clinical investigation of medicinal products in the paediatric population (ICH E11) and all other applicable ethical guidelines. GSK obtained favourable opinion/approval to conduct the study prior to a site initiating the study in that country or has documented that neither a favourable opinion nor an approval to conduct the study was needed. Written informed consent/assent was obtained from subjects or their parents/guardians prior to enrolment. The study received approval from the following ethics committees (ECs), or institutional review boards (IRBs) and regional authorities were consulted consistent with country requirements, namely Commissie Medische Ethiek UZ/ KU Leuven (EC) in Belgium (approval received 24 August); Ethik-Kommission der Bayerischen 
(EC) in Germany (approval received 20 September), and in Spain the PEIBA (IRB and RA combined) for Andalusia (approval received 26 September) and Hospital Vall de Hebron (IRB) and Goli Gurina (RA) for Catalonia (approval received 22 October). GSK posted the study protocol on GSK's Clinical Study Register (accessible at www.gsk-clinicalstudyregister.com, GSK Study ID 207737) and on the US National Library of Medicine website www.clinicaltrials. gov (NCT03688620).

Data Availability. To request access to patient-level data and documents for this study, please submit an enquiry via www. clinicalstudydatarequest.com.

Open Access. This article is distributed under the terms of the Creative Commons Attribution-NonCommercial 4.0 International License (http://creativecommons.org/licenses/ by-nc/4.0/), which permits any noncommercial use, distribution, and reproduction in any medium, provided you give appropriate credit to the original author(s) and the source, provide a link to the Creative Commons license, and indicate if changes were made.

\section{REFERENCES}

1. Cassini A, Colzani E, Pini A, et al. Impact of infectious diseases on population health using incidence-based disability-adjusted life years (DALYs): results from the Burden of Communicable Diseases in Europe study, European Union and European Economic Area countries, 2009 to 2013. Euro Surveill. 2018;23(16).

2. World Health Organisation (WHO). Fact Sheets. Influenza (seasonal). http://www.who.int/newsroom/fact-sheets/detail/influenza-(seasonal). Accessed 1 Apr 2019.

3. World Health Organization (WHO). Influenza vaccines. http://www.who.int/influenza/vaccines/en/. Accessed 1 Apr 2019.

4. European Medicines Agency (EMA). Interim guidance on enhanced safety surveillance for seasonal influenza vaccines in the EU 2014. http://www. ema.europa.eu/docs/en_GB/document_library/
Scientific_guideline/2014/04/WC500165492.pdf. Accessed 1 Apr 2019.

5. European Medicines Agency (EMA). Explanatory note on the withdrawal of the Note for guidance on harmonisation of requirements for influenza vaccines and of the core SmPC/PL for inactivated seasonal influenza vaccines. 2014. https://www.ema. europa.eu/en/documents/scientific-guideline/ explanatory-note-withdrawal-note-guidanceharmonisation-requirements-influenza-vaccinescore-summary_en-0.pdf. Accessed 1 Apr 2019.

6. De Lusignan S, Correa A, Dos Santos G, et al. Brand specific, near real time, enhanced safety surveillance of influenza vaccines in general practice winter 2015/16: feasibility study. JMIR Public Health Surveill. 2019;10:12016.

7. de Lusignan S, Ferreira F, Damaso S, et al. Enhanced passive surveillance of influenza vaccination in England, 2016-2017: an observational study using an adverse events reporting card. Human Vaccines Immunother. 2019;15(5):1048-59.

8. de Lusignan S, Dos Santos G, Byford R, et al. Enhanced safety surveillance of seasonal quadrivalent influenza vaccines in english primary care: interim analysis. Adv Ther. 2018;35(8):1199-214.

9. Medicines.org.uk. Package leaflet. Fluarix Tetra suspension for injection in pre-filled syringe 2018. https://www.medicines.org.uk/emc/files/pil.3021. pdf. Accessed 1 Apr 2019.

10. Dos Santos G, Yeakey A, Shende V, et al. Passive enhanced safety surveillance of GSK's quadrivalent seasonal influenza vaccine in Belgium, Germany and Spain, an observational study: protocol for the 2018/2019 influenza season. BMJ Open. 2019;9(8):e028043.

11. Medical Dictionary for Regulatory Activities MedDRA. https://www.meddra.org/basics. Accessed 1 Apr 2019.

12. European Centre for Disease Prevention and Control (ECDC). Seasonal influenza vaccines 2019. https://ecdc.europa.eu/en/seasonal-influenza/ prevention-and-control/seasonal-influenza-vaccines. . Accessed 12 Sept 2019.

13. Korn EL, Graubard BI. Confidence intervals for proportions with small expected number of positive counts estimated from survey data. Survey Methodol. 1998;24(2):193-201.

14. Medicines.org.uk. Fluarix Tetra Summary of Product Characteristics (SmPC). 2018. https://www. medicines.org.uk/emc/product/3021/smpc. Accessed 18 May 2019. 
15. Spila Alegiani S, Alfonsi V, Appelgren EC, et al. Active surveillance for safety monitoring of seasonal influenza vaccines in Italy, 2015/2016 season. BMC Public Health. 2018;18(1):1401.

16. van Balveren-Slingerland L, Kant A, Harmark L. Web-based intensive monitoring of adverse events following influenza vaccination in general practice. Vaccine. 2015;33(19):2283-8.

17. Dos Santos G. Challenges in implementing yearly enhanced safety surveillance of influenza vaccination in Europe: lessons learned and future perspectives. Human Vaccines Immunother. 2019;22:1-13. 